\title{
Wave dispersion and attenuation in viscoelastic split Hopkinson pressure bar
}

\author{
Z.Q. Cheng, J.R. Crandall and W.D. Pilkey \\ Department of Mechanical, Aerospace and Nuclear \\ Engineering, University of Virginia, Charlottesville, \\ VA 22903-2442, USA
}

A viscoelastic split Hopkinson pressure bar intended for testing soft materials with low acoustic impedance is studied. Using one-dimensional linear viscoelastic wave propagation theory, the basic equations have been established for the determination of the stress-strain-strain rate relationship for the tested material. A method, based on the spectral analysis of wave motion and using measured wave signals along the split Hopkinson pressure bar, is developed for the correction of the dispersion and attenuation of viscoelastic waves. Computational simulations are performed to show the feasibility of the method.

Keywords: Split Hopkinson pressure bar, viscoelastic wave, dispersion, attenuation, spectral analysis

\section{Introduction}

The split Hopkinson pressure bar (SHPB) is one of the best available and most widely accepted techniques for the determination of the stress-strain characteristics of materials at high stain rates [6-8]. A basic SHPB consists of a striker bar, an incident bar and a transmitter bar, as shown in Fig. 1. The specimen to be tested is placed between the incident and transmitter bars. Usually, both incident and transmitter bars are made of the same high-strength elastic materials, such as steel or aluminium. While the specimen of the tested material, usually of lower strength, may deform non-elastically, the bars remain in an elastic state. Therefore, it is generally assumed that elastic waves propagate through the incident and transmitter bars.

According to the theory of wave propagation in solids [9], the strength of the transmitted wave signal depends on the difference between the acoustic impedance $\rho C_{0}$ (where $\rho$ is the density of the material and $C_{0}$ is wave velocity) of the bars and the tested material. The greater the difference between them, the weaker the transmitted wave signal. If the acoustic impedance of the tested material is much lower than that of bars, the transmitted wave signal will be too weak to be measured with sufficient accuracy. Therefore, the difference between the acoustic impedance of bars and the material to be tested should be kept within a reasonable range. For instance, the SHPB made of aluminium alloys or other materials with $\rho C_{0}$ of the order of $10 \mathrm{MPa} / \mathrm{m}$ was successfully used in the studies of the nonlinear viscoelastic behavior of engineering plastics with $\rho C_{0}$ of the order of $1 \mathrm{MPa} \mathrm{s} / \mathrm{m}$ at high strain rates, but was not suitable for testing materials with $\rho C_{0}$ of the order of $0.1 \mathrm{MPa} / \mathrm{m}$ or lower [13]. In order to test soft materials with low acoustic impedances at high strain rates by the SHPB, it has been suggested [13] that the bars be made of viscoelastic materials, such as polymers, which have relatively low acoustic impedance.

\section{Problems with viscoelastic SHPB}

For the specimen tested using the SHPB, as shown in Fig. 1, the average strain rate, strain, and stress can be expressed as

$$
\begin{aligned}
\dot{\varepsilon}_{\mathrm{s}}(t) & =\frac{v\left(x_{\mathrm{s} 2}, t\right)-v\left(x_{\mathrm{s} 1}, t\right)}{l_{\mathrm{s}}} \\
& =\frac{v_{\mathrm{t}}\left(x_{\mathrm{s} 2}, t\right)-v_{\mathrm{i}}\left(x_{\mathrm{s} 1}, t\right)-v_{\mathrm{r}}\left(x_{\mathrm{s} 1}, t\right)}{l_{\mathrm{s}}}, \\
\varepsilon_{\mathrm{s}}(t) & =\int_{0}^{t} \dot{\varepsilon}_{\mathrm{s}}(t) \mathrm{d} t \\
& =\frac{1}{l_{\mathrm{s}}} \int_{0}^{t}\left[v_{\mathrm{t}}\left(x_{\mathrm{s} 2}, t\right)-v_{\mathrm{i}}\left(x_{\mathrm{s} 1}, t\right)-v_{\mathrm{r}}\left(x_{\mathrm{s} 1}, t\right)\right] \mathrm{d} t, \\
\sigma_{\mathrm{s}}(t) & =\frac{A}{2 A_{\mathrm{s}}}\left[\sigma\left(x_{\mathrm{s} 1}, t\right)+\sigma\left(x_{\mathrm{s} 2}, t\right)\right] \\
& =\frac{A}{2 A_{\mathrm{s}}}\left[\sigma_{\mathrm{i}}\left(x_{\mathrm{s} 1}, t\right)+\sigma_{\mathrm{r}}\left(x_{\mathrm{s} 1}, t\right)+\sigma_{\mathrm{t}}\left(x_{\mathrm{s} 2}, t\right)\right],
\end{aligned}
$$




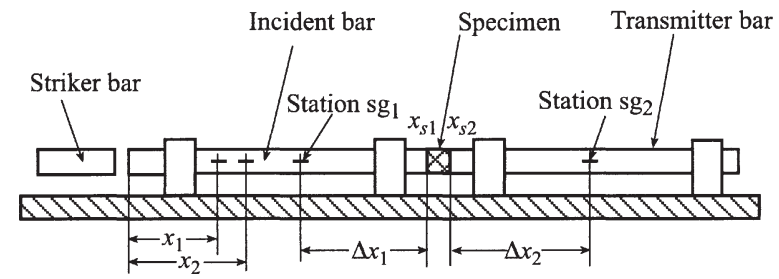

Fig. 1. Schematic diagram of the SHPB.

where $l_{\mathrm{s}}$ is the length of the specimen; $A$ is the cross sectional area of incident and transmitter bars; $A_{\mathrm{s}}$ is the cross sectional area of the specimen; $v\left(x_{\mathrm{s} 1}, t\right)$ and $v\left(x_{\mathrm{s} 2}, t\right)$ are the particle velocities at the left and right interfaces of the specimen; $\sigma\left(x_{\mathrm{s} 1}, t\right)$ and $\sigma\left(x_{\mathrm{s} 2}, t\right)$ are the stresses at the interfaces; and the subscripts i, r, and $\mathrm{t}$ denote the corresponding quantities of incident, reflected, and transmitted waves, respectively.

For the elastic SHPB, assume that a longitudinal elastic wave propagates through the bars, and define that the incident pulse and transmitted pulse are forward waves and the reflected pulse is a backward wave. Then

$$
\begin{aligned}
& v_{\mathrm{i}}\left(x_{\mathrm{s} 1}, t\right)=-C_{0} \varepsilon_{\mathrm{i}}\left(x_{\mathrm{s} 1}, t\right), \\
& v_{\mathrm{t}}\left(x_{\mathrm{s} 2}, t\right)=-C_{0} \varepsilon_{\mathrm{t}}\left(x_{\mathrm{s} 2}, t\right), \\
& v_{\mathrm{r}}\left(x_{\mathrm{s} 1}, t\right)=C_{0} \varepsilon_{\mathrm{r}}\left(x_{\mathrm{s} 1}, t\right) .
\end{aligned}
$$

Here $C_{0}=\sqrt{E / \rho_{0}}$, where $E$ is the Young's modulus and $\rho_{0}$ is the density of the bar material; and

$$
\begin{aligned}
\sigma\left(x_{\mathrm{s} 1}, t\right) & =\sigma_{\mathrm{i}}\left(x_{\mathrm{s} 1}, t\right)+\sigma_{\mathrm{r}}\left(x_{\mathrm{s} 1}, t\right) \\
& =E\left[\varepsilon_{\mathrm{i}}\left(x_{\mathrm{s} 1}, t\right)+\varepsilon_{\mathrm{r}}\left(x_{\mathrm{s} 1}, t\right)\right], \\
\sigma\left(x_{\mathrm{s} 2}, t\right) & =\sigma_{\mathrm{t}}\left(x_{\mathrm{s} 2}, t\right)=E \varepsilon_{\mathrm{t}}\left(x_{\mathrm{s} 2}, t\right) .
\end{aligned}
$$

Substituting Eqs (2) and (3) into Eq. (1) leads to the conventional equations for the elastic SHPB [8]:

$$
\begin{aligned}
& \dot{\varepsilon}_{\mathrm{s}}(t)=\frac{C_{0}}{l_{0}}\left(\varepsilon_{\mathrm{i}}-\varepsilon_{\mathrm{r}}-\varepsilon_{\mathrm{t}}\right), \\
& \varepsilon_{\mathrm{s}}(t)=\frac{C_{0}}{l_{\mathrm{s}}} \int_{0}^{t}\left(\varepsilon_{\mathrm{i}}-\varepsilon_{\mathrm{r}}-\varepsilon_{\mathrm{t}}\right) \mathrm{d} t, \\
& \sigma_{\mathrm{s}}(t)=\frac{E A}{2 A_{\mathrm{s}}}\left[\varepsilon_{\mathrm{i}}+\varepsilon_{\mathrm{r}}+\varepsilon_{\mathrm{t}}\right] .
\end{aligned}
$$

Usually, these strains are not measured directly at the interfaces. Instead, they are measured by strain gages placed away from the specimen, as shown in Fig. 1, where $\varepsilon_{\mathrm{i}}$ and $\varepsilon_{\mathrm{r}}$ are measured by the strain gage at station $\mathrm{sg}_{1}$, and $\varepsilon_{\mathrm{t}}$ is measured by the strain gage at station $\mathrm{sg}_{2}$. This is considered to be acceptable pro- vided that the ratio of the bar radius to the wave length does not exceed $0.1[2,3,12]$. However, a longitudinal wave is dispersive. A wave, or a stress pulse, will be distorted as it travels along the bars. Therefore, some methods have been proposed for the correction of the dispersion of waves in the elastic SHPB [3-5,11,15].

There are two significant differences in the use of the viscoelastic SHPB relative to the use of the elastic SHPB. One is that as a viscoelastic wave propagates through the bars, it will attenuate and disperse [10], so that the stresses and particle velocities at the two interfaces of the specimen cannot be directly determined from the measurements of strain stations away from the specimen. The correction of the dispersion and attenuation is necessary for the use of a viscoelastic SHPB. Another problem is that since Eqs (2) and (3) are not valid for viscoelastic waves, Eq. (4) can not be used to calculate the stress-strain-strain rate relationship for the tested material. While Eq. (1), as a fundamental equation describing the stress-strain-strain rate relationship for the specimen, is valid for the viscoelastic SHPB, it is necessary to establish a relationship for the specimen which connects the measured strains with the stresses and particle velocities.

To assist in the use of SHPB with viscoelastic bars, an analytical method was employed [13] to address the two questions. The method was based on a constitutive equation of viscoelastic material and characteristics theory of one-dimensional linear viscoelastic wave propagation. However, there are some limitations with this approach.

- Although the constitutive relationships for viscoelastic materials were represented by a three parameter model (standard model), which can be considered as a Maxwell model with an auxiliary spring in parallel as shown in Fig. 2, it is only able to describe the mechanical properties of viscoelastic materials over a very narrow frequency range [10].

- As pointed out for the elastic SHPB, the longitudinal wave is dispersive. One-dimensional linear viscoelastic wave propagation theory on which the approach was based does not account for the dispersion of the longitudinal wave.

- Support and boundary conditions of the bars, which were not taken into account in the approach, may contribute to wave dispersion and attenuation [1].

An alternative approach, using a spectral analysis, will be employed in this paper to develop a method for 


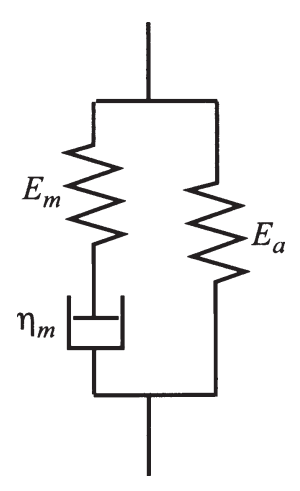

Fig. 2. Maxwell model with an auxiliary spring in parallel.

the generalization of the SHPB from elastic bars to viscoelastic bars. The development of this method begins with one-dimensional linear viscoelastic wave propagation theory. This method is, however, experimentally based.

\section{Dispersion and dissipation of viscoelastic waves in SHPB}

\subsection{Linear viscoelastic wave propagation equation}

Since the bar deformation in SHPB should be small, the characteristic of the bar material can be described by a one-dimensional linear constitutive law [10]

$$
\sigma=E_{0} \varepsilon+\int_{-\infty}^{t} \bar{\psi}(t-\tau) \frac{\partial \varepsilon}{\partial \tau} \mathrm{d} \tau
$$

where $\sigma$ is the one-dimensional stress; $\varepsilon$ is the onedimensional strain; $E_{0}$ is referred to as the "instantaneous" modulus and is related to Lamè's constants $\lambda$ and $\mu$; and $\bar{\psi}(t)$ is a memory function or stress relaxation function with the properties

$$
\begin{aligned}
& \bar{\psi}(t)=0 \quad \text { if } t \leqslant 0, \\
& \lim _{t \rightarrow \infty} \bar{\psi}(t)=0 .
\end{aligned}
$$

The equation of motion for a thin bar is

$$
\rho_{0} \frac{\partial^{2} u}{\partial t^{2}}=\frac{\partial \sigma}{\partial x}
$$

where $u=u(x, t)$ is the displacement of the particles at the position of $x$. The relationship between the strain $\varepsilon$ and the displacement $u(x, t)$ is

$$
\varepsilon=\frac{\partial u(x, t)}{\partial x} .
$$

Thus,

$$
\frac{\partial v}{\partial x}=\frac{\partial \varepsilon}{\partial t}
$$

where $v$ is the velocity of the particles at $x$, and

$$
v=\frac{\partial u(x, t)}{\partial t}
$$

Substituting Eqs (5) and (8) into Eq. (7) leads to

$$
C_{0}^{2} \frac{\partial^{2} u}{\partial x^{2}}+\int_{-\infty}^{t} \bar{\psi}_{0}(t-\tau) \frac{\partial^{3} u}{\partial \tau \partial x^{2}} \mathrm{~d} \tau=\frac{\partial^{2} u}{\partial t^{2}}
$$

where $C_{0}=\sqrt{E_{0} / \rho_{0}}$, and $\bar{\psi}_{0}(t)=\bar{\psi}(t) / \rho_{0}$. This is the one-dimensional linear viscoelastic wave propagation equation [14].

Apply the Fourier transform to this equation, giving

$$
\left[C_{0}^{2}+\mathrm{i} \omega \bar{\Psi}_{0}(\omega)\right] \frac{\partial^{2} U(\omega)}{\partial x^{2}}=(\mathrm{i} \omega)^{2} U(\omega),
$$

where

$$
\begin{aligned}
\bar{\Psi}(\omega) & =F\left[\bar{\psi}_{0}(t)\right], \\
U(\omega) & =F[u(t)] .
\end{aligned}
$$

Here $F[\cdot]$ denotes the Fourier transform.

Define

$$
B^{2}(\omega)=\frac{(i \omega)^{2}}{C_{0}^{2}+\mathrm{i} \omega \bar{\Psi}_{0}(\omega)},
$$

then

$$
\frac{\partial^{2} U(\omega)}{\partial x^{2}}=B^{2}(\omega) U(\omega)
$$

Thus

$$
U(\omega)=A \mathrm{e}^{ \pm B(\omega) x}
$$

where $A$ is the amplitude spectrum, and the minus sign corresponds to forward waves and the plus sign to backward waves. Denote

$$
B(\omega)=\alpha(\omega)+\mathrm{i} k(\omega)
$$

where $k$ is the wave number related to wave dispersion and $\alpha$ is the attenuation factor related to wave attenu- 
ation. Both of them depend on material properties and are functions of frequency $\omega$. That is, $k=k(\omega), \alpha=$ $\alpha(\omega)$. The particle displacement $u(x, t)$ can be obtained from Eq. (16) using the inverse Fourier transform

$$
u(x, t)=F^{-1}[U(\omega)]=F^{-1}\left[A \mathrm{e}^{ \pm B(\omega) x}\right]
$$

where $F^{-1}[\cdot]$ denotes the inverse Fourier transform. For a monochromatic wave,

$$
u(x, t)=A \mathrm{e}^{ \pm B(\omega)} \mathrm{e}^{\mathrm{i} \omega t} .
$$

\subsection{Basic relations for viscoelastic SHPB}

One of advantages of using the elastic SHPB is that only strain signals need to be measured and these can be easily recorded by strain gages located away from the specimen. This advantage should be retained for the viscoelastic SHPB. For the viscoelastic SHPB, Eqs (2) and (3) no longer hold, so that Eq. (4) is not applicable.

From Eqs (5), (8), (10) and (16),

$$
\begin{aligned}
E(x, \omega) & = \pm B(\omega) U(x, \omega) \\
& = \pm A B(\omega) \mathrm{e}^{ \pm B(\omega) x},
\end{aligned}
$$

and

$$
\begin{aligned}
& \Sigma(x, \omega)=\frac{-\rho_{0} \omega^{2}}{B^{2}(\omega)} E(x, \omega), \\
& V(x, \omega)=\mp \frac{\mathrm{i} \omega}{B(\omega)} E(x, \omega),
\end{aligned}
$$

where in the signs of \pm and $\mp$, the minus sign corresponds to forward waves and the plus sign to backward waves. Here

$$
\begin{aligned}
& E(x, \omega)=F[\varepsilon(x, t)], \\
& \Sigma(x, \omega)=F[\sigma(x, t)], \\
& V(x, \omega)=F[v(x, t)] .
\end{aligned}
$$

The inverse Fourier transform of this equation gives $\varepsilon(x, t), \sigma(x, t)$, and $v(x, t)$.

Equation (20) describes the dispersion and attenuation relations of strain signals. Using this equation, the strains at the interfaces of the specimen can be determined from those measured at strain stations away from the specimen. Then, using Eq. (21), the stresses and particle velocities at the interfaces can be ob- tained, and the stress-strain-strain rate relationship of the tested material can be determined by Eq. (1).

Theoretically, if the constitutive law of the bar material, as described by Eq. (5), is known, the function $B(\omega)$ of Eq. (14), or, equivalently, the attenuation factor $\alpha(\omega)$ and wave number $k(\omega)$ can be determined, and hence the correction of the dispersion and attenuation of stress waves propagating in the viscoelastic SHPB can be accomplished. However, as has been stated previously, there are some problems with the function $B(\omega)$ when it is determined theoretically. Therefore, in this paper, with the basic relations for viscoelastic SHPB being described by Eqs (1) and (21), the determination of the function $B(\omega)$, i.e., the correction of the dispersion and attenuation of viscoelastic waves in the SHPB, is treated by a more general method.

\section{Correction of dispersion and attenuation by spectral analysis}

\subsection{Spectral analysis of viscoelastic waves}

According to the theory of spectral analysis of wave motion, a one-dimensional wave can be expressed by its spectrum decomposition form [1]

$$
\begin{aligned}
u(x, t) & =\sum_{n} F_{n}\left\{G_{1 n}+G_{2 n}+\cdots\right\} \mathrm{e}^{\mathrm{i} \omega_{n} t} \\
& =\sum_{n} F_{n} G_{n} \mathrm{e}^{\mathrm{i} \omega_{n} t}
\end{aligned}
$$

where $G_{j n}(j=1,2, \ldots)$ is a transfer function of the $j$ th mode, a function of position $x$ and frequency $\omega_{n}$; $F_{n}$ is the amplitude spectrum, which is known from the input conditions or from some measurement. Thus, $F_{n} G_{n}$ is recognized as the Fourier transform of the wave. It is generally assumed that only the first mode is excited in longitudinal wave propagation. Therefore,

$$
u(x, t)=\sum_{n} F_{n} G_{1 n} \mathrm{e}^{\mathrm{i} \omega_{n} t} .
$$

For one-dimensional linear viscoelastic wave propagation, from Eqs (16) and (17),

$$
G_{1 n}=\mathrm{e}^{-\left(\alpha_{n}+\mathrm{i} k_{n}\right) x},
$$

where only the forward wave is considered. 


\subsection{Identification of wave number and attenuation factor}

Suppose at $x_{1}$ and $x_{2}$, the measured wave signals are $u\left(x_{1}, t\right)$ and $u\left(x_{2}, t\right)$, respectively. Using a fast Fourier transform (FFT), their corresponding spectrums are

$$
\begin{aligned}
& U_{1}(\omega)=\operatorname{FFT}\left[u\left(x_{1}, t\right)\right]=\sum_{n} U_{1 n} \mathrm{e}^{\mathrm{i} \phi_{1 n}}, \\
& U_{2}(\omega)=\operatorname{FFT}\left[u\left(x_{2}, t\right)\right]=\sum_{n} U_{2 n} \mathrm{e}^{\mathrm{i} \phi_{2 n}} .
\end{aligned}
$$

From Eqs (24) and (25), we have the following relations:

$$
\begin{aligned}
& U_{1 n} \mathrm{e}^{\mathrm{i} \phi_{1 n}}=F_{n} \mathrm{e}^{-\left(\alpha_{n}+\mathrm{i} k_{n}\right) x_{1}}, \\
& U_{2 n} \mathrm{e}^{\mathrm{i} \phi_{2 n}}=F_{n} \mathrm{e}^{-\left(\alpha_{n}+\mathrm{i} k_{n}\right) x_{2}} .
\end{aligned}
$$

Then, the attenuation factor $\alpha_{n}$ and wave number $k_{n}$ can be identified from the measured wave signals at $x_{1}$ and $x_{2}$

$$
\hat{\alpha}_{n}=\frac{\ln U_{1 n}-\ln U_{2 n}}{x_{2}-x_{1}},
$$

and

$$
\hat{k}_{n}=\frac{\phi_{1 n}-\phi_{2 n}}{x_{2}-x_{1}}
$$

However, since $\phi_{1 n}$ and $\phi_{2 n}$ come from the Fourier transform of the wave signals, it is probable that

$$
\begin{aligned}
& \phi_{1 n}=\phi_{1 n}^{\prime}+2 l_{1} \pi, \\
& \phi_{2 n}=\phi_{2 n}^{\prime}+2 l_{2} \pi,
\end{aligned}
$$

where $\phi_{1 n}^{\prime}$ and $\phi_{2 n}^{\prime}$ are the original phases of the components; and $l_{1 n}$ and $l_{2 n}$ are integers. Substituting Eq. (30) into Eq. (29) yields

$$
\hat{k}_{n}=\frac{\phi_{1 n}^{\prime}-\phi_{2 n}^{\prime}+2\left(l_{1 n}-l_{2 n}\right) \pi}{x_{2}-x_{1}} .
$$

Let

$$
k_{n}=\frac{\phi_{1 n}^{\prime}-\phi_{2 n}^{\prime}}{x_{2}-x_{1}}
$$

be the original value of the wave number. In general, $l_{1 n} \neq l_{2 n}$, so that $\hat{k}_{n} \neq k_{n}$. This is a problem of the loss of phase information due to the Fourier transform. To solve this problem, it was suggested that the values of $\left(l_{1 n}-l_{2 n}\right)$ must be chosen so that Eq. (31) gives a reasonable phase velocity and frequency relationship [15]. However, this condition is difficult to employ in practical computations.

However, if we let

$$
x=l \cdot \Delta x,
$$

where $x$ is the position of the wave to be corrected or predicted, $l$ is an integer, and $\Delta x=x_{2}-x_{1}$, then, according to Eqs (31) and (32), the identified wave number $\hat{k}_{n}$ can be used to replace the original wave number $k_{n}$ for the purposes of the correction of the dispersion.

If more than two stations are available, the least square estimate of $\hat{\alpha}_{n}$ is

$$
\begin{aligned}
& \hat{\alpha}_{n}=\left[\left\{x_{k}-x_{j}\right\}^{\mathrm{T}}\left\{x_{k}-x_{j}\right\}\right]^{-1} \\
& \quad \times\left\{x_{k}-x_{j}\right\}^{\mathrm{T}}\left\{\ln U_{j n}-\ln U_{k n}\right\}, \\
& \{j=1,2, \ldots, M-1 ; k=j+1, j+2, \ldots, M\},
\end{aligned}
$$

where $M$ is the number of stations.

\subsection{Correction procedures}

1. Use a proper time window and sampling rate to digitize the wave signal from each strain gage station. Impose the FFT on the digitized wave signal to obtain its frequency spectrum.

2. Identify the attenuation factor and wave number for each frequency component of the wave using Eqs (28) or (34), and (29).

3. Suppose the wave at the position of $x_{\mathrm{c}}$ needs to be corrected or predicted from the measured wave at the position of $x_{\mathrm{r}}$. Construct the frequency spectrum of the wave to be corrected or predicted using the following equation

$$
U_{\mathrm{c}}(x, \omega)=\sum_{n} U_{\mathrm{r} n} \mathrm{e}^{\mathrm{i} \phi_{\mathrm{r} n}} \mathrm{e}^{-\left(\alpha_{n}+\mathrm{i} k_{n}\right) \Delta x_{\mathrm{c}}},
$$

where

$$
\Delta x_{\mathrm{c}}=x_{\mathrm{c}}-x_{\mathrm{r}},
$$

and $U_{\mathrm{r} n} \mathrm{e}^{\mathrm{i} \phi_{\mathrm{r} n}}$ is obtained from the FFT of the wave signal at $x_{\mathrm{r}}$.

4. Obtain the corrected or predicted wave signal using the IFFT

$$
u_{\mathrm{c}}(x, t)=\operatorname{IFFT}\left[U_{\mathrm{c}}(x, \omega)\right] .
$$




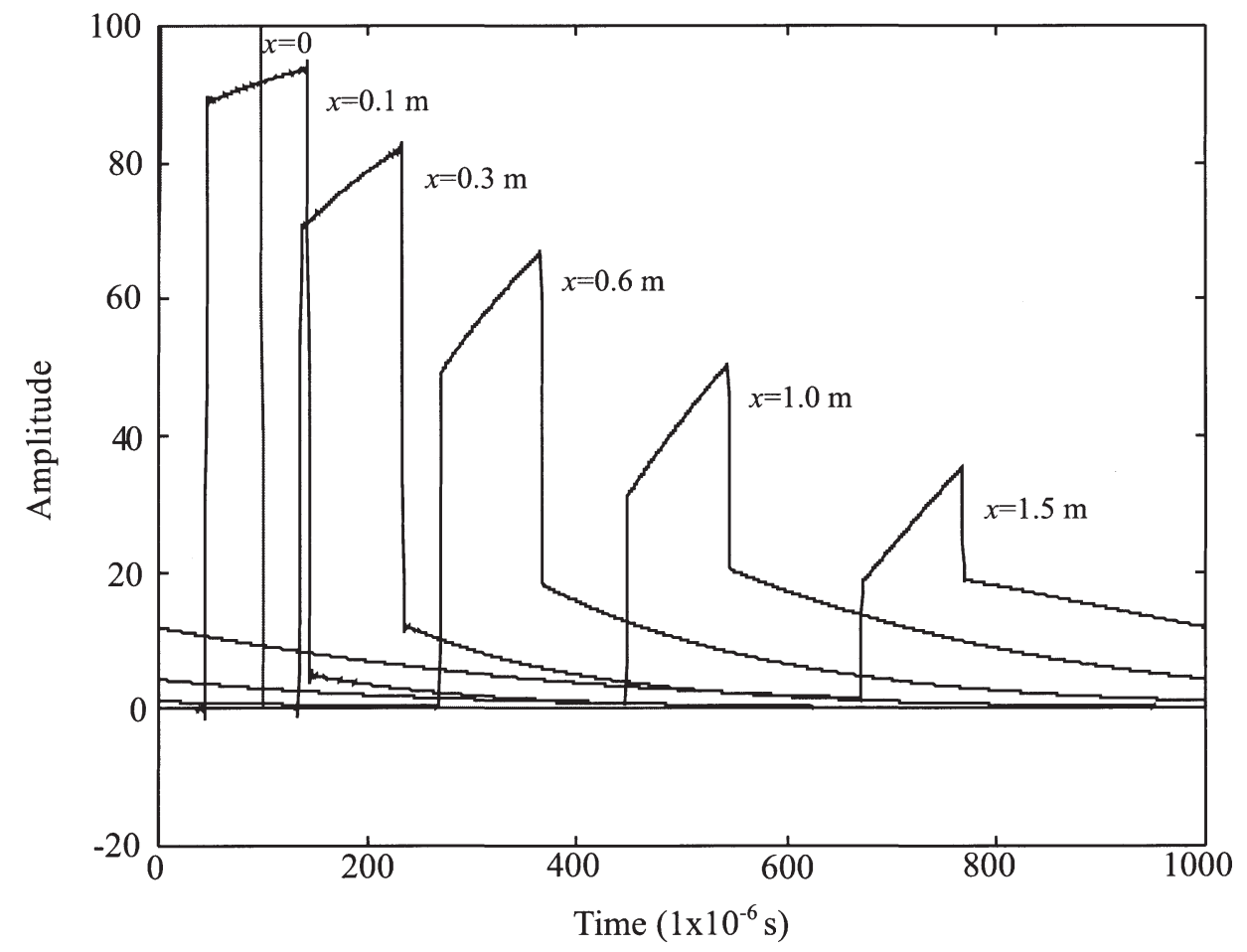

Fig. 3. Propagation of viscoeleastic waves.

\section{Numerical simulations}

Both the incident and transmitted bars are made of plastic. Its characteristics can be described by the Maxwell model with a spring in parallel, as shown in Fig. 2, having the parameters $E_{\mathrm{a}}=3.14 \mathrm{GPa}, E_{\mathrm{m}}=$ $3.98 \mathrm{GPa}, \eta_{\mathrm{m}}=0.268 \mathrm{MPa}$ and $\rho=1.2 \times 10^{3} \mathrm{~kg} / \mathrm{m}^{3}$.

The wave number $k$ and attenuation factor $\alpha$ can be theoretically determined by the following equations [13]:

$$
\begin{aligned}
k^{2}= & \frac{\rho \omega^{2}}{2 E_{\mathrm{a}}}\left\{\left[\frac{1+\omega^{2} \theta_{2}^{2}}{1+\left(1+E_{\mathrm{m}} / E_{\mathrm{a}}\right)^{2} \omega^{2} \theta_{2}^{2}}\right]^{1 / 2}\right. \\
& \left.+\frac{1+\left(1+E_{\mathrm{m}} / E_{\mathrm{a}}\right) \omega^{2} \theta_{2}^{2}}{1+\left(1+E_{\mathrm{m}} / E_{\mathrm{a}}\right)^{2} \omega^{2} \theta_{2}^{2}}\right\},
\end{aligned}
$$

and

$$
\begin{aligned}
\alpha^{2}= & \frac{\rho \omega^{2}}{2 E_{\mathrm{a}}}\left\{\left[\frac{1+\omega^{2} \theta_{2}^{2}}{1+\left(1+E_{\mathrm{m}} / E_{\mathrm{a}}\right)^{2} \omega^{2} \theta_{2}^{2}}\right]^{1 / 2}\right. \\
& \left.-\frac{1+\left(1+E_{\mathrm{m}} / E_{\mathrm{a}}\right) \omega^{2} \theta_{2}^{2}}{1+\left(1+E_{\mathrm{m}} / E_{\mathrm{a}}\right)^{2} \omega^{2} \theta_{2}^{2}}\right\},
\end{aligned}
$$

where $\theta_{2}=\eta_{\mathrm{m}} / E_{\mathrm{m}}$.

A pulse of trapezoidal shape is generated at the input end of the incident bar, which is shown in Fig. 3 $(x=0)$. The duration of this pulse is $T_{0}=100 \mu \mathrm{s}$. To sample the pulse, choose sampling rate $\Delta t=0.9766 \mu \mathrm{s}$ and time window $T=1000 \mu \mathrm{s}$. The size of the time window is chosen so as not only to allow proper characterization of the spectral content of the pulse, but also to allow room for propagation of the signal [1]. The FFT is employed for a spectral analysis of the signals. According to the sampling parameters of the pulse, the fundamental frequency, i.e., the frequency increment, is $\omega_{0}=6280 \mathrm{rad} / \mathrm{s}$.

From Eqs (37) and (38), the relationships of the wave number and attenuation factor versus each frequency component are shown in Figs 4(a) and (b), respectively. As the pulse propagates forward through the viscoelastic bars, it will be dispersed and attenuated. Using the dispersion and attenuation relations described by Eqs (37), (38) and shown in Figs 4(a) and (b), the waves at the positions of $x=$ $[0.1,0.3,0.6,1.0,1.5] \mathrm{m}$ are calculated, as shown in Fig. 3. These waves can be considered as the waves predicted form the initial pulse, the wave at $x=0$.

Now, suppose the wave signals shown in Fig. 3 are the measured strain signals at the corresponding strain 


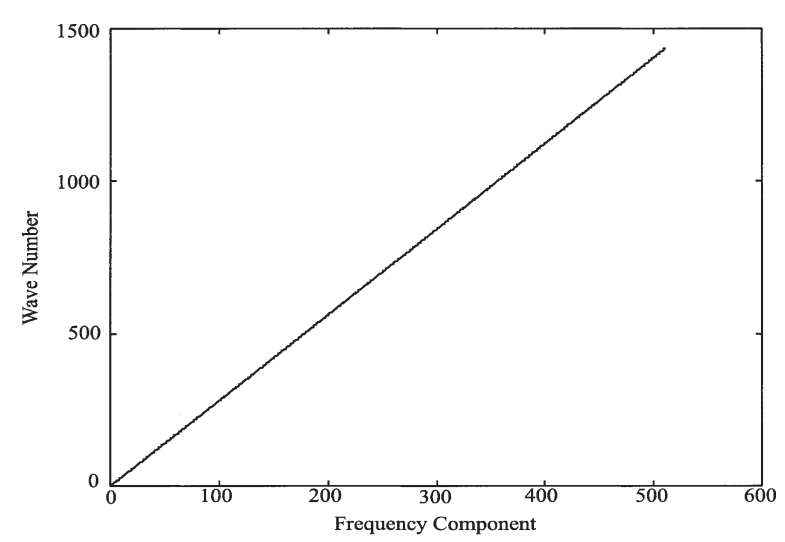

(a)

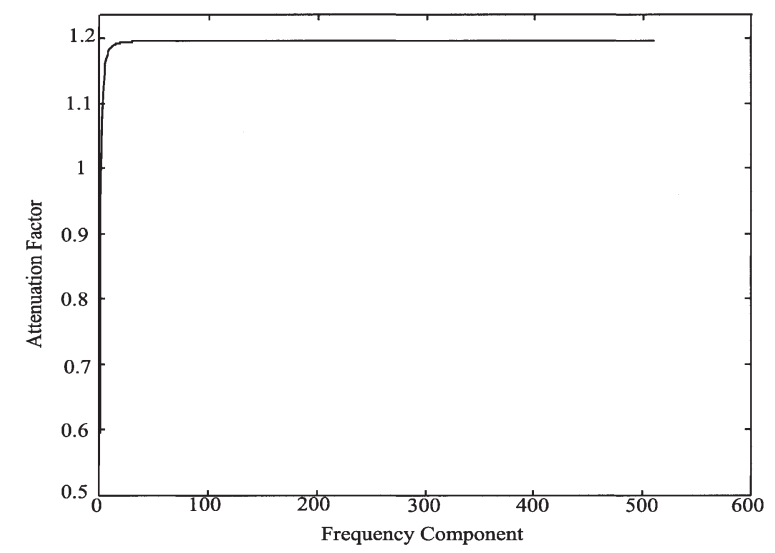

(b)

Fig. 4. Theoretical wave numbers and attenuation factors.

gage stations. The wave number and attenuation factor can be identified from these measurements. Using Eq. (29), wave numbers are identified, with the results shown in Fig. 5(a) for the case when the signals at $x=0.3 \mathrm{~m}$ and $x=0.6 \mathrm{~m}$ are considered. Using Eq. (34), attenuation factors are identified which are shown in Fig. 5(b). It can be seen by comparing Fig. 4 and Fig. 5 that the attenuation factors identified are nearly identical to those theoretically calculated, but the wave numbers identified are very different from those theoretically calculated. This, as stated previously, is the result of the loss of phase information during the FFT. Moreover, if signals from different stations are used in the identification, different identified wave numbers will result.

Using identified wave numbers and attenuation factors, the waves at $x=0, x=0.6 \mathrm{~m}$, and $x=1.5$ are corrected or predicted from the measured wave at $x=0.3 \mathrm{~m}$, as shown in Fig. 6. Comparing them with those in Fig. 3 at the same positions, we find that as soon as the condition of Eq. (33) is satisfied, the at-

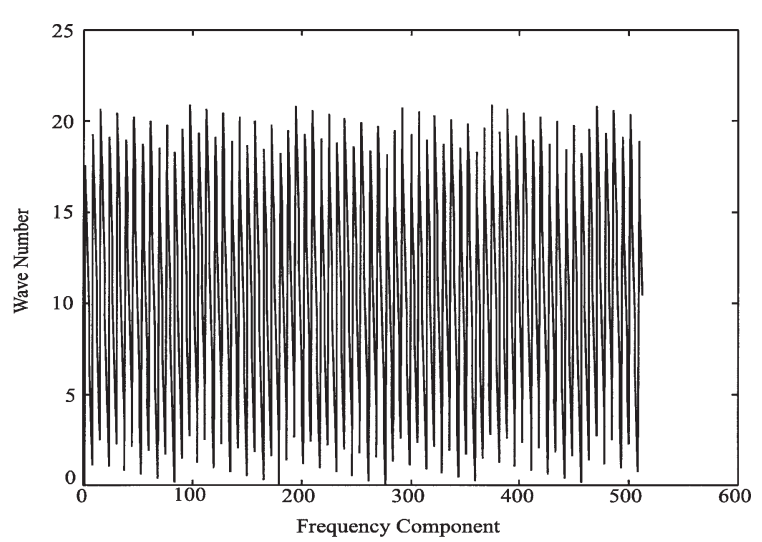

(a)

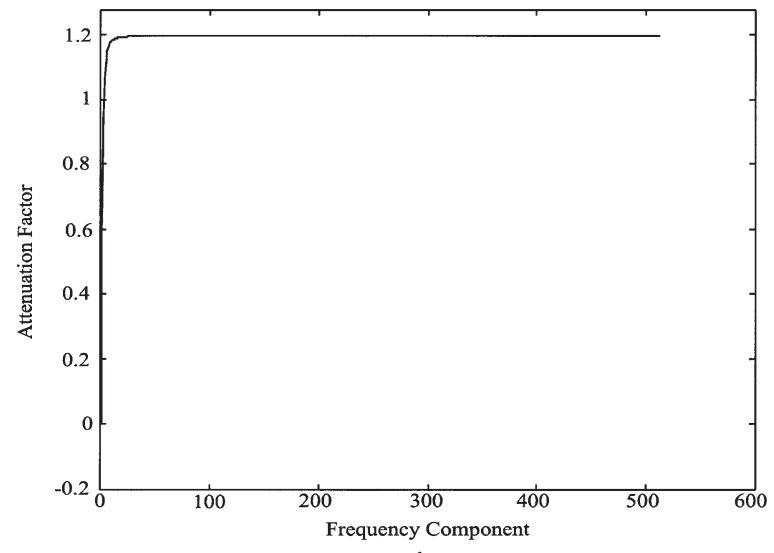

(b)

Fig. 5. Identified wave numbers and attenuation factors.

tenuated and dispersed waves can be corrected or predicted accurately, though the identified wave numbers are different from their real ones. But, if the condition of Eq. (33) is not satisfied, the corrected or predicted waves will be severely distorted. Such an example is shown in Fig. 7, where the wave at $x=1.0 \mathrm{~m}$ is predicted from the measured wave at $x=0.3 \mathrm{~m}$ using identified wave numbers and attenuation factors. The real shape of this wave is shown in Fig. 3 for $x=1.0 \mathrm{~m}$.

\section{Concluding remarks}

The generalization of the SHPB from elastic bars to viscoelastic bars has been investigated. The main problem with this generalization is related to the attenuation and dispersion of viscoelastic waves. A method for the correction of the dispersion and attenuation of the viscoelastic waves in the SHPB is developed based on the spectral analysis of wave motion. From the analy- 


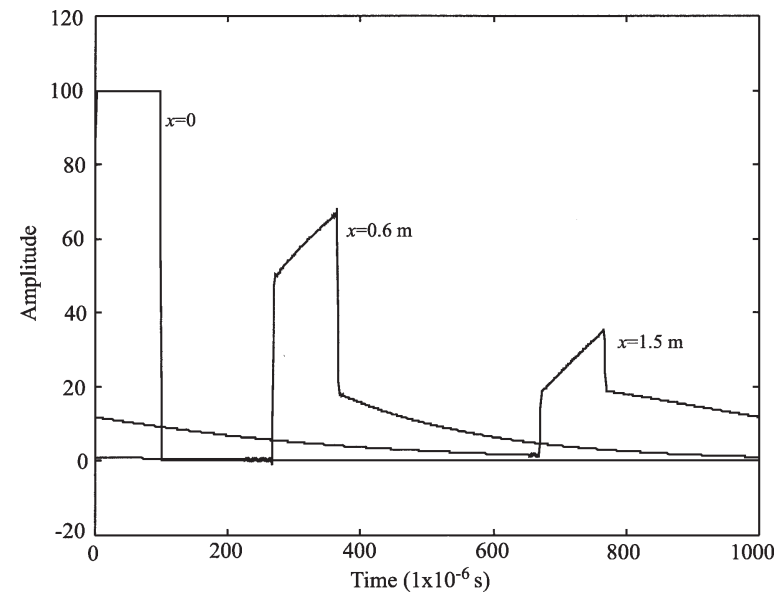

Fig. 6. The corrected and predicted waves.

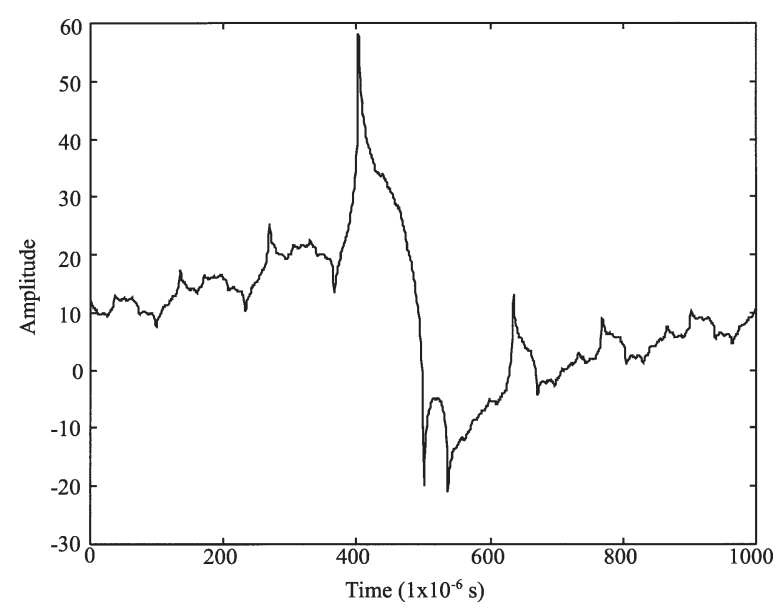

Fig. 7. An incorrect predicted wave.

ses and computational simulation, the following conclusions are in order.

1. The generalization of the SHPB from the elastic bars to the viscoelastic bars permits the testing of soft material with low acoustic impedance.

2. The main advantages of the elastic SHPB can be retained for the viscoelastic SHPB. Among them is the contention that the stress-strain-strain rate relationship of the tested material can still be obtained from the measured strains at stations away from the specimen.

3. The correction of the dispersion and attenuation of the viscoelastic waves in the SHPB can be accomplished using identified wave numbers and attenuation factors. It is not necessary to know the bar material constitutive properties. With a spectral analysis of measured wave signals, not only the material dispersion, but the geometrical dispersion as well can be corrected.

4. The measurement stations should be appropriately positioned, in order to counter the effects of the loss of phase information due to the FFT in the identified wave numbers.

This paper is concerned primarily with the problem of the correction of the dispersion and attenuation of waves in the viscoelastic SHPB. There are some other difficulties with the application of the viscoelastic SHPB, such as the effect of the rate-dependent property of viscoelastic material, and the influence of the noise in the measured signals on the identification.

\section{References}

[1] J.F. Doyle, Wave Propagation in Structures - An FFT-Based Spectral Analysis Methodology, Springer-Verlag, New York, 1989.

[2] R.M. Davies, A critical study of the Hopkinson pressure bar, Philosophical Transactions A 240 (1948), 375-457.

[3] P.S. Follansbee and C. Frantz, Wave propagation in the split Hopkinson pressure bar, J. Engng. Materials and Tech. 105 (1983), 61-66.

[4] D.A. Gorham, A numerical method for the correction of dispersion in pressure bar signal, J. Phys. E: Sci. Instrum. 6 (1983), 477-479.

[5] J.C. Gong, L.E. Malvern and D.A. Jenkins, Dispersion investigation in the split Hopkinson pressure bar, J. of Engng. Materials and Tech. 112 (1990), 309-314.

[6] L.J. Griffiths, D.J. Parry and R.P. Worthington, A comparison of optical and strain gauge techniques in the determination of the dynamic mechanical behavior of carbon-fibre composite using a split Hopkinson Pressure bar, in: Proc. 2nd Conf. on the Mechanical Properties of Materials at High Rates of Strain, J. Harding, ed., Oxford, England, 1979, pp. 28-30.

[7] J. Harding, The effects of high strain rate on material properties, Chapter 4 in: Materials at High Strain Rates, T.Z. Blazynski, ed., Elsevier Applied Science, 1987.

[8] U.S. Lindholm, Some experiments with split Hopkinson pressure bar, J. Mech. Phys. Solids 12 (1964), 317-335.

[9] H. Kolsky, Stress Waves in Solids, Clarendon, Oxford, 1953.

[10] H. Kolsky, Viscoelastic waves, in: Int. Symposium on Stress Wave Propagation in Material, Norman Davids, ed., Interscience, London, 1960.

[11] J.M. Lifshitz and H. Leber, Data processing in the split Hopkinson pressure bar tests, Int. J. Impact Engng. 15(6) (1994), 723-733.

[12] G. Ravichandran and G. Subhash, Critical appraisal of limiting strain rates for compression testing of ceramics in a split Hopkinson pressure bar, J. Am. Ceram. Soc. 77(1) (1994), 263-267.

[13] L. Wang, K. Labibes, Z. Azari and G. Pluvinage, Generalization of split Hopkinson bar technique to use viscoelastic bars, Int. J. Impact Engng. 15(5) (1994), 669-686. 
[14] S.T. Xue, J. Tobita, T. Hanzawa and M. Izumi, Wave attenuation in viscoelastic continuum with fading memory, J. of Engng. Mech. 118(8) (1991), 1597-1611.
[15] E.H. Yew and C.S. Chen, Experimental study of dispersive waves in beam and rod using FFT, J. of Appl. Mech. 45 (1978), 940-942. 

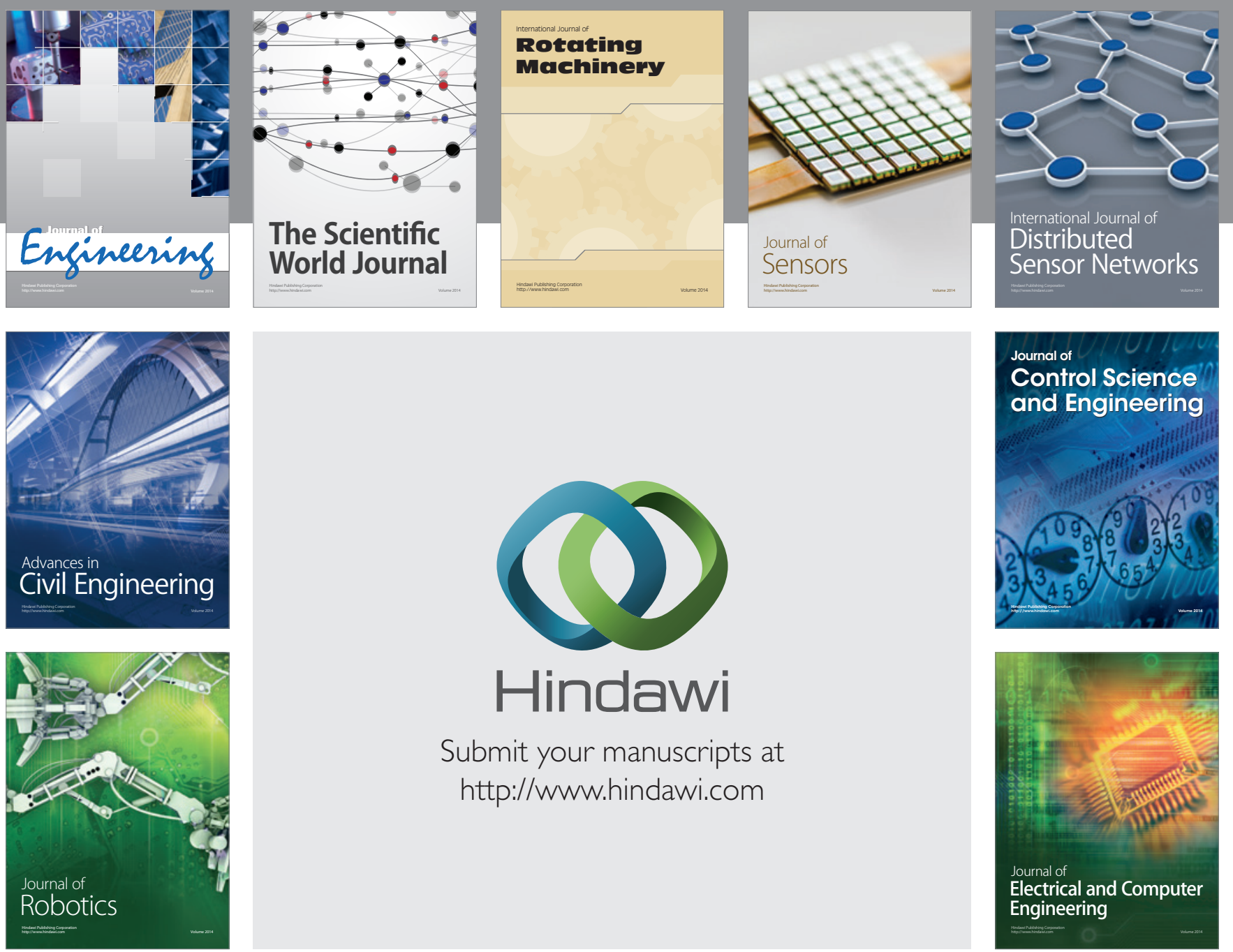

Submit your manuscripts at

http://www.hindawi.com
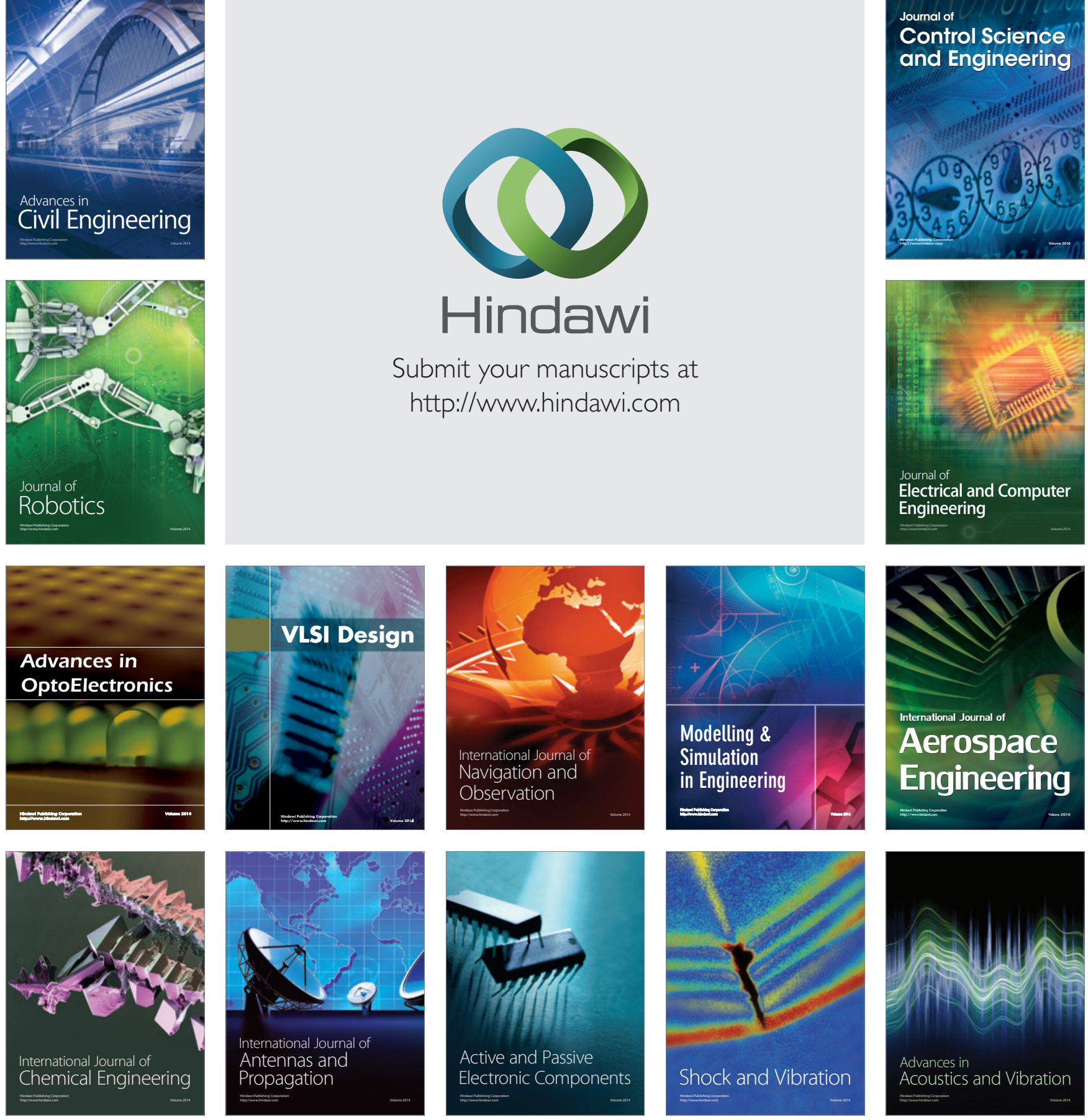